\title{
Models of Care for musculoskeletal pain conditions: driving change to improve outcomes
}
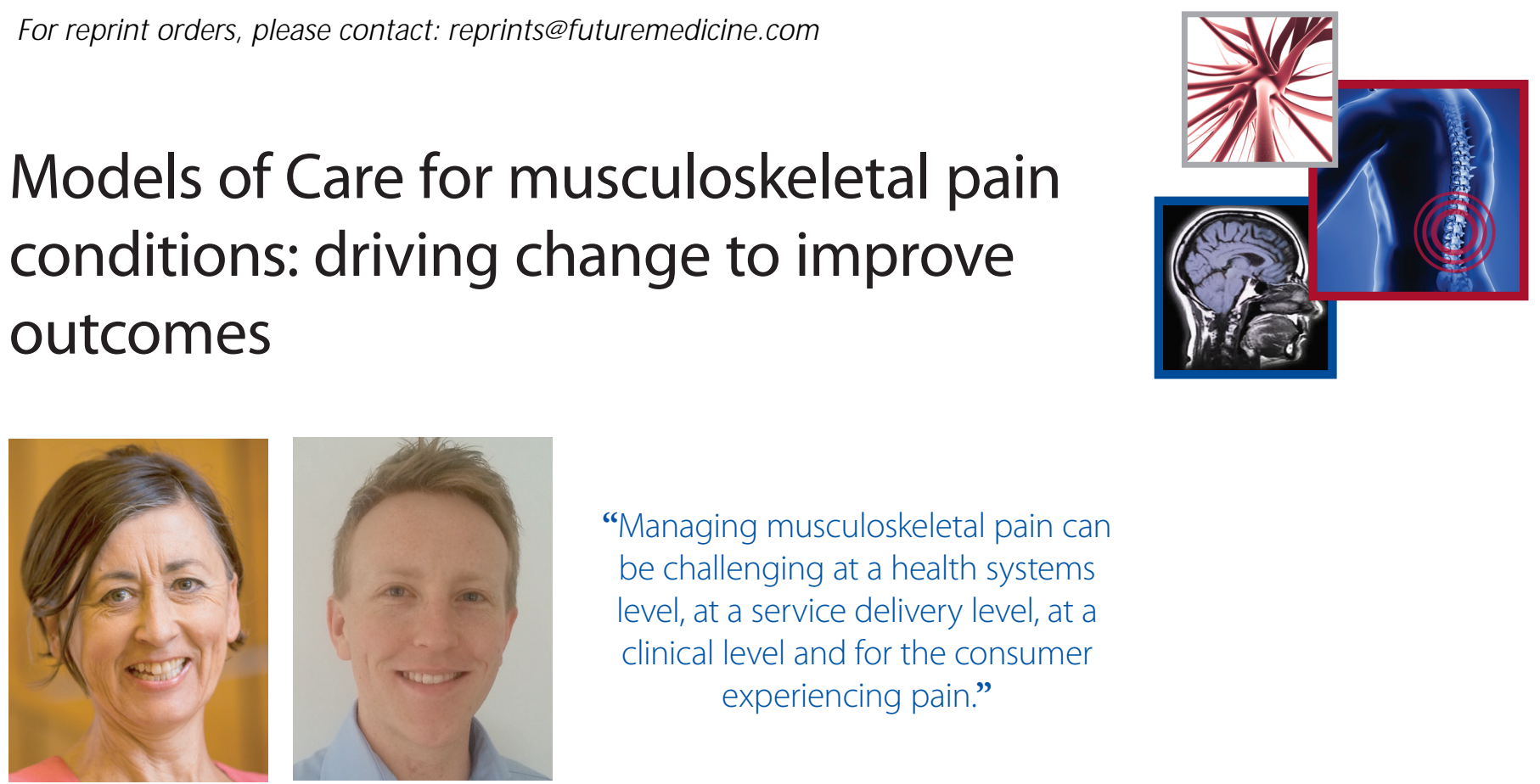

\author{
"Managing musculoskeletal pain can \\ be challenging at a health systems \\ level, at a service delivery level, at a \\ clinical level and for the consumer \\ experiencing pain.”
}

Helen Slater ${ }^{* 1,2} \&$ Andrew M Briggs ${ }^{1}$

First draft submitted: 3 April 2017; Accepted for publication: 30 May 2017; Published online: 22 September 2017

\begin{abstract}
Musculoskeletal pain \& models of care
Managing musculoskeletal pain can be challenging at a health systems level, at a service delivery level, at a clinical level and for the consumer experiencing pain. Approaching this complexity to both facilitate person-centered care and respond to the burden associated with musculoskeletal pain in an efficient, effective and sustainable way, requires reform reaching across policy and into clinical practice. In this context, Models of Care (MoCs) are increasingly regarded as a system-level enabler to translate evidence for 'what works' from policy/ systems, across service delivery and into practice. MoCs provide a platform for a reform agenda in health systems by describing not only what care to deliver but also 'how to' deliver care. We have previously comprehensively reviewed the evidence for musculoskeletal MoCs [1]. In the following commentary, rather than a focus on MoCs per se, we use working examples to show how the implementation of musculoskeletal pain MoCs is being enabled to support reform agendas. We make an explicit distinction between a
\end{abstract}

MoC which provides principle-level guidance on what care to deliver and how to implement it, and a Model of Service Delivery (MoSD) which operationalizes these principles into local activity, informed by an implementation plan [2].

\section{- MoCs are needed for improved} musculoskeletal pain management

Pain management for musculoskeletal conditions is inadequate in much of the world. There are four key reasons: limited access to appropriate treatments for pain; a broad failure to recognize that persistent or chronic pain is a serious chronic health problem requiring access to integrated management similar to other chronic diseases such as diabetes; significant knowledge and skills deficiencies of health care professionals, consumers and the broader community regarding the understanding, assessment and management of acute and persistent pain from a contemporary pain science perspective; and finally, many countries lack a national policy agenda regarding the management of pain as a health priority, either in its own right or part of

ISchool of Physiotherapy \& Exercise Science, Curtin University, GPO Box U1987, Perth, WA 6845, Australia

${ }^{2}$ School of Medicine, The University of Notre Dame, Fremantle, Perth, Australia

*Author for correspondence: Tel.: + 6189266 3099; Fax: + 6189266 3699; H.Slater@curtin.edu.au 
a broader noncommunicable disease approach. In this context, MoCs have a key role to play as a system-level vehicle to drive reform and help translate evidence for 'what works' in musculoskeletal pain management, into policy and clinical practice.

\section{- The escalating \& non-sustainable health \&} economic burden of musculoskeletal pain Musculoskeletal pain is one of the most common reasons that people seek medical help. However, in many cases, pain still goes underrecognized and under-treated, despite access to appropriate and evidence-based pain care being acknowledged as a fundamental human right [3]. Globally, the health and economic burdens associated with musculoskeletal pain are substantial, both at a societal and health consumer level [4-8]. Chronic or persistent pain has broad reach, affecting the lives of one in five people globally, including children, with musculoskeletal pain contributing a significant proportion of this burden [9-11]. Recent Global Burden of Disease data point to an increasing burden (disability-adjusted life years) of noncommunicable diseases, particularly musculoskeletal conditions [12]. By condition, low back and neck pain are the leading cause of disability in both developed and developing countries, with the disability burden associated with osteoarthritis increasing substantially between 2005 and 2015 [12]. When considering morbidity and mortality combined, low back pain is ranked fourth [12]. Future projections for the burden of disease associated with musculoskeletal conditions highlight risks associated with workforce and sustainability issues. A recent Australian socioeconomic impact report has estimated a massive $43 \%$ increase in prevalence in cases of musculoskeletal pain-related conditions by 2032 [4]. In this context, addressing the burden of disease for musculoskeletal pain-related conditions across economies and across the life course through the development of appropriate policy and service responses (such as MoCs), is an urgent global priority [13].

\section{- Contemporary best practice in musculoskeletal pain management}

The adoption of a biopsychosocial approach is consistently recommended as best practice pain management [8-10]. Such an approach provides a useful clinical framework for addressing the whole person, acknowledging the dynamic, multidimensional nature of pain and making sense of the variable and often misconstrued relationship between pain severity, physical pathology findings and disability [14]. Multidimensional domains are ideally addressed using multimodal approaches ideally tailored to complexity (stratified by risk of complexity/chronicity/disability). Contemporary MoSDs include components of care such as self-management strategies, psycho-education (neurophysiology of pain), use of active strategies such as pacing (time-contingent graded activity), behavioral approaches to pain (for example, addressing catastrophizing, distress, unhelpful beliefs, fear-avoidance behaviors), active movement-based strategies, combined with pharmacological and interventional procedural options, as appropriate $[8,10]$. An integrative biopsychosocial MoSD is ideally tailored to the needs of the individual [1] and is delivered within a health system that measures outcomes in a systematized, robust way [15]. This in no way implies that such a MoSD belongs solely to the domain of tertiary care, although for some people with complex pain problems associated with high levels of disability, including co- or multimorbidities, this setting may be most appropriate. Rather, many pain problems can be managed effectively within primary care settings, especially where appropriate system-level MoCs have been implemented [1].

Despite advances in many areas of pain medicine, health outcomes remain variable [10]. While a recent systematic analysis of all evidence-based treatments for acute pain has demonstrated recent improvements in safety and effectiveness, significant gaps persist between evidence and practice [16]. Similarly for people with chronic pain, only modest improvements in long term outcomes are achieved at best [10]. Additional challenges occur at the individual level, where people living with chronic musculoskeletal pain often confront stigma. Many face a daily battle to find an explanation for their pain and prove legitimacy, and are subjected to navigating complex health systems and contexts that do not support helpful beliefs or management practices related to their condition (e.g., compensation settings) [17]. The personal impact of ongoing musculoskeletal pain is further amplified by an increase in prevalence of common co- and multimorbidities such as anxiety, depression and sleep disorders with associated negative impacts on their productivity and quality of life [12]. Obtaining 
timely, effective, evidence-informed and integrated care that captures these comorbidities is challenging, especially given that the complexity of musculoskeletal pain is typically poorly understood across the community, by educators, researchers and trainee and trained health professionals alike $[1,17]$.

Implementing musculoskeletal MoCs to support improved pain management

Complex problems like pain need efficient and effective systems approaches at a health systems level (macro), at a service delivery level (meso) and at an individual consumer and clinician level (micro). This multilevel challenge lends itself well to the use of an $\mathrm{MoC}$ as a vehicle to support musculoskeletal pain reform [18]. High level advocacy for people with musculoskeletal pain is helping to drive such reform agendas through the implementation of national and pan-nation Pain Strategies [6-8,10]. The alignment of these Pain Strategies and other international pain reform initiatives [19] with musculoskeletal pain MoCs, encourages cross sector engagement and shared decision making, thereby better informing priority areas and supporting effective, sustainable implementation [13]. While the focus of calls to action has come primarily from middle-tohigh income economies, importantly, there are also initiatives that lever off the scalability of eHealth technologies to support implementation of MoCs in lower and middle income economies [20]. Such initiatives help attenuate care disparities and disease burden associated with musculoskeletal pain and other chronic noncommunicable diseases.

Recently, we have extensively reviewed the evidence for $\mathrm{MoC}$ application to musculoskeletal pain conditions [1]. In a further themed series of papers on MoCs [13], we and colleagues have reported on what care should be delivered to people with musculoskeletal painrelated conditions, and also on how to best support implementation of coordinated care, across different economic settings to achieve the right care at the right time in the right place, delivered by the right team, and using the right resources [13]. In this commentary, we constrain our focus to musculoskeletal pain more broadly, rather than to specific musculoskeletal conditions. We provide practical working examples of enablers to implementation of MoCs and outline how these initiatives are being used to support musculoskeletal pain reform through informing policy and health strategy priorities, resourcing and health governance decisions (macro factors); service design and workforce capacity building initiatives (meso factors); and consumers' participation in care and clinicians' practice behaviors (micro factors).

\section{- Macro-level initiatives to support implementation of MoCs}

A key example of a system-level (macro) initiative designed to support the implementation of musculoskeletal MoCs is the use of standardized pain outcomes data captured through centralized registries. Such system-level data capture allows for the monitoring and evaluation of health and economic outcomes in relation to musculoskeletal pain management, thereby informing strategic policy, governance and planning of service delivery priorities.

\section{Use of centralized registries to support} implementation

The use of eHealth-enabled centralized referral systems in public healthcare settings, as a component of an $\mathrm{MoC}$, can help to ensure timely access (right time) to appropriate care (right care) and help to optimize downstream service delivery efficiencies (right resources) [2]. Systems that offer standardized, efficient data entry, and the collation and monitoring of system, clinical and patient-reported outcomes are increasingly needed in order to address the escalating burden associated with musculoskeletal pain conditions. The two following examples of such initiatives highlight how using cross sector partnerships can support effective implementation.

The electronic Persistent Pain Outcomes initiative (ePPOC) [15] is a strategic implementation initiative of the Australian and New Zealand College of Anesthetists, Faculty of Pain Medicine, which aligns with the National Pain Strategy. The initial phase started in 2013, with further cross-sector development by the Faculty of Pain Medicine, the Australian Pain Society and the wider pain community. ePPOC enables a systemized electronic collection of a standardized set of data items and assessment tools by specialist pain services throughout Australia and New Zealand, including capture of person-centered, clinical and system outcomes. This data collection reaches across Australian state jurisdictions and includes pediatric and adult services in both

\author{
"Pain management for \\ musculoskeletal conditions \\ is inadequate in much of \\ the world."
}


"Models of care have a

key role to play as a

system-level vehicle to

drive reform and help

translate evidence for 'what

works' in musculoskeletal

pain management, into

policy and clinical

practice." the public and private sectors. The outcomes of this initiative include system, service and health data [15], potentially allowing for benchmarking of facilities against best practice.

A similar efficient systematized data collection approach has been developed and implemented in the USA, the Collaborative Health Outcomes Information Registry (CHOIR) initiative [21]. CHOIR uses open source, free data-collection software created in partnership with cross-discipline scientists, clinical experts and the National Institutes of Health. CHOIR allows clinicians to capture both quantitative and qualitative information about people with pain (currently $\sim 10,000$ cases) in a safe, secure and easy-to-use system. This enables sophisticated reporting of individual personcentered data against population-derived normative data, in addition to systems level data about clinical pathways and cost effectiveness. Systems such as ePPOC and CHOIR allow for cross-jurisdictional comparison of system, clinical and person-centered pain outcomes, thereby providing important data, which can be utilized for benchmarking of practice and comparative health economic analyses.

\section{- Meso-level initiatives to support implementation of MoCs}

One of the most important rate-limiting factors constraining the delivery of effective pain management is deficiencies in the current and emerging health workforce. Currently, the delivery of care inadequately aligns with best available evidence for what works and care disparities created by geography, socioeconomic status and ethnicity further widen these burden-service gaps. Yet, reliable data indicate that the burden of pain associated with musculoskeletal conditions can be substantially reduced when available evidence-based management is implemented [1] Furthermore, such care is associated with measurable downstream health and economic benefits $[7,22]$. In order to deliver integrated MoSDs that support person-centered care an appropriately skilled health workforce is required. Here, we focus on examples of building system and workforce capacity to support implementation of contemporary musculoskeletal pain MoCs [1].

Building health workforce capacity as an implementation enabler

In advance of the 2018 Global Year of Pain Education, targeted initiatives to support health professional capacity building are currently being undertaken by the International Association for the Study of Pain (IASP). These include a review of current IASP pain curricula (freely accessible on the IASP website) with a focus on embedding knowledge and core competencies that are aligned across disciplines, to better support upskilling of the emerging health workforce. A complementary initiative led by Professor Scott Fishman from UC Davis and colleagues and supported by a grant from the MayDay Foundation, recently attracted leading pain educators from around the world to an invited closed workshop held in Yokohama, Japan. The workshop was focused on advancing global education and core pain competencies through driving change in formal training curricula, and across disciplines [23]. The IASP has a number of additional initiatives supporting workforce capacity building to address the challenges of pain management in resource-limited countries, such as pain training camps in Myanmar for the pain community in Southeast Asia.

eHealth systems can also facilitate workforce capacity building to support implementation of MoCs (for comprehensive review, see Slater et al. [13,20]). There are numerous contemporary initiatives that demonstrate the ways in which workforce capacity can be scaled using eHealth technologies. The TelePain Initiative [24] implemented across a number of states in the USA uses video, web and telephone conferencing technologies to support the delivery of cross-sector pain services. The Extension for Community Health Outcomes (ECHO: specifically, the Ontario Chronic Pain and Opioid Stewardship at UHN [25]) is a telehealth program that has been successfully implemented to support knowledge transfer from 'one to many' primary care providers, thereby building selfefficacy and capacity for improved pain care in Ontario, Canada. ECHO has an extended reach including into New Mexico, thereby dramatically increasing the access to specialty treatments for complex conditions including pain, in rural and underserved areas. The use of innovative telephone- and Internet-delivered model of service delivery for people with osteoarthritis pain through the Australian Centre for Health Exercise and Sports Medicine, shows clinically meaningful improvements in pain and function that are sustained for at least 6 months [26]. Another innovative use of technology to health professional support capacity building, is the 
Body in Mind website [27], a collaborative initiative developed to promote knowledge translation, and improve healthcare professionals' understanding of clinical pain sciences. This e-resource has provided in excess of $600 \mathrm{blog}$ posts (from over 200 authors in 22 countries) with a focus on translating evidence-based pain research into more clinically usable forms. Collectively, such cross-discipline and global initiatives help address some of the current burdenservice gaps in pain and support the sustainable implementation of musculoskeletal MoCs.

The use of triaging \& risk stratification as an implementation enabler

At a service delivery level, efficient systems for triaging of musculoskeletal pain-related conditions can assist with early identification, prevention of chronicity musculoskeletal pain and direction to the appropriate clinical pathways to ensure consumers receive the 'right care, at the right time, by the right team.' Risk stratification (chronicity or disability) can also help guide tiered resource allocation toward those with higher disability who typically require more complex, integrated care. Initiatives using innovative web and smartphonebased multidimensional electronic pain assessment tools (e-Ouch, Standardized Universal Pain Evaluation for Rheumatology providers [SUPERKIDZ] and Pain-QuILTTM for timely triage of children and adolescents with pain) are available for use in primary care and subspecialty clinical settings [11]. The Sheffield Back Pain model focuses on early recognition of risk for progression to chronicity in primary care settings by using a validated risk stratification tool (the STarTBack screening tool). Treatment is matched to the risk of chronicity, with resource allocation supporting lower risk management in primary care settings, and multidisciplinary care for those at higher risk. Similarly, many primary care settings have adopted short-form derivatives of the validated Örebro Musculoskeletal Pain Screening Questionnaire, to help estimate risk of chronicity and guide management decisions and resource allocation, including within compensable settings [17].

Health system inversion as an implementation enabler

Using a system inversion MoSD, aligned with an $\mathrm{MoC}$ in Western Australia, inter-professional consumer group education sessions delivered prior to face-to-face practitioner appointments have resulted in significantly reduced wait-times and costs at public pain medicine units [22]. Further, consumer use of active pain self-management strategies increased with fewer requiring individual consultations. This MoSD is recurrently resourced and has now been adopted in a number of primary care sites throughout Australia [22].

\section{- Micro-level initiatives to support implementation of MoCs}

Building consumer \& clinician capacity to support implementation

Building individual consumer and clinician capacity is critical to supporting sustainable implementation of MoCs. Providing accessible, timely, appropriate pain resources for consumers and their treating clinicians enables shared decision making and encourages helpful behavior change for both groups.

For consumers, evidence-based treatment programs delivered via the internet can support effective management of musculoskeletal conditions including arthritis, osteoporosis, and persistent musculoskeletal pain in children, adolescents and adults $[11,20]$. For example, iPeer2Peer is an innovative, tailored peer-mentorship program for young people that provides behavior change support through modeling and reinforcement by trained peers. This program is enabled through Skype video calls conducted over 8 weeks [11]. Consumer-led development of evidenced-based online resources such as the Pain Tool Kit [28] offers free 24/7 access to practical knowledge and skills to support co-care, bypassing some of the access, travel and cost barriers of face-to-face consultations. Another example of use of digital technologies to support co-care is The Pain Course, an internet-delivered intervention offered by the eCentreClinic. The Pain Course was designed to be suitable for Australian people with a broad range of different persistent pain conditions, and importantly is accessible to those living in rural and remote settings, on referral from their treating primary care physician. The Pain Course has proven highly acceptable to consumers, is time efficient and has accumulating evidence for significant clinical reductions in pain, disability, anxiety and depression levels, and increased patient self-efficacy (see commentary in [20]).

In Australia, the development of evidencebased websites such as pain HEALTH [29] and Pain Management Network website [30] provide additional guidance and support to both consumers and their treating health professionals. These shared resources have been informed by 
musculoskeletal MoCs with both websites codesigned in partnership with consumers and healthcare providers, to enable patients to develop practical pain skills and knowledge. Importantly, such cross-sector partnerships have been important to ensuring sustainable implementation of these resources. Implementation has been further assisted through wider dissemination via links to consumer advocacy groups (e.g., Arthritis Australia and painAustralia) and through implementation partners, including professional organizations such as Australian Pain Society and the Australian Physiotherapy Association.

Balancing the escalating burden of musculoskeletal pain against the benefits and risks of providing scalable, evidence-based e-resources for consumers and their treating health professionals will require careful examination of health system, clinical and cost effectiveness outcomes [20]. In this context, the role of eHealth technologies poses an appealing lever to support scalable and sustainable implementation of musculoskeletal MoCs, however rigorous evaluation must be undertaken to ensure improved health outcomes and person-centered outcomes and concurrently identify risk and harm profiles.

\section{Models of care for musculoskeletal pain:} monitoring \& evaluation

While emerging evidence suggests that MoCs are effective in improving system efficiencies and health outcomes [1,13], continued evaluation is important to substantiate their utility and maintain a focus on prioritizing their implementation. As MoCs necessarily have a system-level focus, traditional effectiveness evaluation designs, such as clinical trials, are not always the most appropriate for evaluation and alternative pragmatic designs are required. MoC evaluation needs to consider short and long-term outcomes that are system relevant, inclusive of health economics. This approach to evaluation aligns more closely with policy and health systems research; an evaluation field increasingly accepted as critical to improving health systems [31]. Recently, we have developed a Framework to guide the development, implementation and evaluation of MoCs [32]. The Framework was informed by 93 experts from 30 nations and publicly supported by 54 international organizations [32]. The Framework provides a guide for evaluation of MoCs and suggests appropriate domains for evaluation in Models of Service Delivery.

\section{Conclusion}

Improving outcomes for musculoskeletal pain management matters: for the individual consumer, for their treating clinicians, for the services that deliver care and for the health systems that support care. MoCs can help drive critical health reform and support streamlined, sustainable delivery of evidence-based pain management through MoSDs. Initiatives that build health systems, health workforce and consumer capacity are important enablers to the sustainable implementation of such MoCs. The contextualization of MoCs allows for the alignment of varying needs across developing and developed economies, with evaluation providing valuable data to minimize threats to sustainability and to inform strategic priorities across macro, meso and micro levels of the heath system.

Financial \& competing interests disclosure The authors have no relevant affiliations or financial involvement with any organization or entity with a financial interest in or financial conflict with the subject matter or materials discussed in the manuscript. This includes employment, consultancies, honoraria, stock ownership or options, expert testimony, grants or patents received or pending, or royalties.

No writing assistance was utilized in the production of this manuscript.

\section{Open access}

This work is licensed under the Attribution-NonCommercial-NoDerivatives 4.0 Unported License. To view a copy of this license, visit http://creativecommons.org/ licenses/by-nc-nd/4.0/

\section{References}

Papers of special note have been highlighted as:

- of interest; $\bullet$ of considerable interest

1 Speerin R, Slater H, Li L et al. Moving from evidence to practice: models of care for the prevention and management of musculoskeletal conditions. Best Pract. Res. Clin. Rheumatol. 28(3), 479-515 (2014).
-. This review of Models of Care for musculoskeletal pain conditions outlines health system and implementation strategies used to improve person-centred outcomes and health system performance.

2 Briggs AM, Bragge P, Slater H, Chan M, Towler SC. Applying a Health Network approach to translate evidence-informed policy into practice: a review and case study on musculoskeletal health. BMC Health Serv. Res. 12, 394 (2012).

- This article describes the use of a Health Network framework as a mechanism to facilitate the translation of evidence into 
policy and practice with a focus on musculoskeletal health.

3 International Pain Summit of the International Association for the Study of Pain. Declaration of Montreal: Declaration of Montreal: declaration that access to pain management is a fundamental human right. J. Pain Palliat. Care Pharmacother. 25(1), 29-31 (2011).

4 Arthritis and Osteoporosis Victoria. A Problem Worth Solving. Arthritis and Osteoporosis Victoria, Elsternwick, Victoria (2013).

5 MBF Foundation. The High Price of Pain: The Economic Impact of Persistent Pain in Australia. MBF Foundation, Sydney, Australia (2007).

6 IOM (Institute of Medicine) Relieving Pain in America: A Blueprint for Transforming Prevention, Care, Education and Research. The National Academies Press, Washington, DC (2011).

- This seminal report provides a broad overview of the causes, impact, prevalence, and scope of pain as a public health problem and delineates a direction and priorities for achieving meaningful reform.

7 Societal Impact of Pain Group. Societal Impact of Pain Focus Group Report, European Federation of IASP Chapters. Societal Impact of Pain Group, Belgium (2013).

8 Breivik H, Eisenberg E, O’Brien T, Openminds. The individual and societal burden of chronic pain in Europe: the case for strategic prioritisation and action to improve knowledge and availability of appropriate care. BMC Public Health, 13, 1229 (2013).

- This article highlights the need for strategic prioritization and co-ordinated actions to address the unacceptable and unnecessary burden of chronic pain that plagues European communities and economies.

9 Portenoy RK, Zeltzer L. A Call to Revolutionize Chronic Pain Care in America: An Opportunity in Health Care Reform. The Mayday Fund, NY, USA (2009)

10 Australian and New Zealand College of Anaesthetists. National Pain Strategy. Australian and New Zealand College of Anaesthetists, Faculty of Pain Medicine, Melbourne (2010).

11 Stinson J, Connelly M, Kamper SJ, Herlin T, Toupin April K. Models of Care for addressing chronic musculoskeletal pain and health in children and adolescents. Best Pract. Res. Clin. Rheumatol. 30(3), 468-482 (2016).

- This article provides a contemporary review of current best practice, focusing on both the 'what' and 'how' best to deliver care to address chronic pain in children and young people.

12 G. B. D. DALYs and Hale Collaborators. Global, regional, and national disabilityadjusted life-years (DALYs) for 315 diseases and injuries and healthy life expectancy (HALE), 1990-2015: a systematic analysis for the Global Burden of Disease Study 2015. Lancet 388(10053), 1603-1658 (2016).

13 Briggs AM, Chan M, Slater H. Models of Care for musculoskeletal health: moving towards meaningful implementation and evaluation across conditions and care settings. Best Pract. Res. Clin. Rheumatol. 30(3), 359-374 (2016).

- This review prefaces a special journal edition on Models of Care and highlights not only what care should be delivered for musculoskeletal conditions, but also 'how' to effectively implement this care.

14 Slater H, Jordan JE, Chua J, Schutze R, Wark JD, Briggs AM. Young people's experiences of persistent musculoskeletal pain, needs, gaps and perceptions about the role of digital technologies to support their co-care: a qualitative study. BMJ Open, 6(12), e014007 (2016).

15 Tardif H, Arnold C, Hayes C, Eagar K. Establishment of the Australasian electronic persistent pain outcomes collaboration. Pain Med.18(6), 1007-1018 (2017).

16 Schug SA, Palmer GM, Scott DA, Halliwell $\mathrm{R}$, Trinca J. Acute pain management: scientific evidence (fourth edition). Med. J. Aust. 204(8), 315-317 (2016).

17 Beales D, Fried K, Nicholas M, Blyth F, Finniss D, Moseley GL. Management of musculoskeletal pain in a compensable environment: implementation of helpful and unhelpful Models of Care in supporting recovery and return to work. Best Pract. Res. Clin. Rheumatol. 30(3), 445-467 (2016).

18 Briggs AM, Jordan JE, Jennings $\mathrm{M}$ et al. Supporting evaluation and implementation of musculoskeletal Models of Care: a globallyinformed framework for judging 'readiness' and 'success'. Arthritis Care Res.69(4), 567-577 (2017).

19 International Association For The Study Of Pain. National, Regional, and Global Pain Initiatives. www.iasp-pain.org

20 Slater H, Dear BF, Merolli MA, Li LC, Briggs AM. Use of eHealth technologies to enable the implementation of musculoskeletal Models of Care: evidence and practice. Best
Pract. Res. Clin. Rheumatol. 30(3), 483-502 (2016).

- This article provides a contemporary review of the use of digital technologies to support the effective implementation of Models of Care for musculoskeletal pain conditions.

21 Stanford University. The Collaborative Health Outcomes Information Registry. https://choir.stanford.edu/

22 Davies S, Quintner J, Parsons R et al. Preclinic group education sessions reduce waiting times and costs at public pain medicine units. Pain Med. 12(1), 59-71 (2011).

23 UC Davis. Advancing Pain Relief Education: Anchoring Pain Competencies Around the World.

www.ucdmc.ucdavis.edu

24 University of Washington. https://depts.washington.edu/anesth

25 Project ECHO. www.echoontario.ca/Echo-Clinic

26 Bennell KL, Nelligan R, Dobson F et al. Effectiveness of an internet-delivered exercise and pain-coping skills training intervention for persons with chronic knee pain: a randomized trial. Ann. Intern. Med. 166(7), 453-462 (2017).

27 Body in Mind Organisation. www.bodyinmind.org/

28 Moore P. The pain toolkit. www.paintoolkit.org/

29 Government of Western Australia, Department of Health. https://painhealth.csse.uwa.edu.au/

30 Agency for Clinical Innovation NG, Health. Pain Management Network. www.aci.health.nsw.gov.au/chronic-pain

31 Ghaffar A, Langlois EV, Rasanathan K, Peterson S, Adedokun L, Tran NT. Strengthening health systems through embedded research. Bull World Health Organ. 95(2), 87 (2017).

32 Briggs AM, Jordan JE, Jennings $M$ et al. A Framework to Evaluate Musculoskeletal Models of Care. Global Alliance for Musculoskeletal Health of the Bone and Joint Decade, Cornwall (2016).

-. This paper describes a Framework (developed by a team of Australian researchers, in partnership with the Global Alliance for Musculoskeletal Health) to help those tasked with planning, implementing or evaluating health services to achieve an optimal Model of Care and its sustainable implementation. 